\title{
Breaking news: DNA damage and repair pathways in COPD and implications for pathogenesis and treatment
}

\author{
Ian M. Adcock ${ }^{1}$, Sharon Mumby ${ }^{1}$ and Gaetano Caramori $\mathbb{1}^{2}$ \\ Affiliations: ${ }^{1}$ Airways Disease Section, National Heart \& Lung Institute, Imperial College, London, UK. ${ }^{2}$ Unità \\ Operativa Complessa di Pneumologia, Dipartimento di Scienze Biomediche, Odontoiatriche e delle Immagini \\ Morfologiche e Funzionali (BIOMORF) Università degli Studi di Messina, Messina, Italy.
}

Correspondence: Ian M. Adcock, Airways Disease Section, Guy Scadding Building, National Heart \& Lung Institute, Imperial College London, Dovehouse Street, London, SW3 6LY, UK.

E-mail: ian.adcock@imperial.ac.uk

@ERSpublications

Defective DNA damage and repair processes are important in COPD

http://ow.ly/edRb30lTF2J

Cite this article as: Adcock IM, Mumby S, Caramori G. Breaking news: DNA damage and repair pathways in COPD and implications for pathogenesis and treatment. Eur Respir J 2018; 52: 1801718 [https://doi.org/ 10.1183/13993003.01718-2018].

Cigarette smoke exposure is the major driver of chronic obstructive pulmonary disease (COPD) and lung cancer in the developed world [1]. COPD affects over 450 million people worldwide whilst lung cancer is the biggest cause of death in people with cancer $[2,3]$. Cigarette smoke-mediated DNA damage causes apoptosis, cellular senescence, inflammation and mutagenesis, which have all been implicated in the pathogenesis of COPD [4,5]. Reduced components of DNA damage and repair pathways have previously been reported in COPD lung tissues (with or without associated pulmonary emphysema) and blood (including stem cells), and airway epithelium and lung fibroblast primary cells [6-10]. A failure to correctly repair cigarette smoke-induced DNA damage has also been reported in COPD [8] and a genetic link to COPD susceptibility noted $[9,11]$. Evidence has also implicated DNA damage and repair mechanisms with lung cancer and response to therapy $[12,13]$. However, the precise mechanisms of DNA damage and repair that are associated with these diseases are unclear.

Several mechanisms for DNA damage and repair exist $[14,15]$, these include: the direct repair pathway which reverses covalently modified nucleotides; base excision repair that repairs incorrect or damaged bases; mismatch repair which repairs aberrant nucleotide insertions or deletions; and nucleotide excision repair (NER) which repairs regions that cause stalling of RNA polymerase. In addition, double stranded breaks are repaired by homologous recombination or non-homologous end-joining. Finally, the Fanconi anaemia pathway integrates multiple DNA repair pathways to repair interstrand cross-links.

Until now there has been no systematic analysis of DNA damage and repair pathways in COPD lung tissue. SAULER et al. [16] used a hypothesis-based bioinformatic approach to determine aberrant expression of 419 selected DNA repair and recombination (DRR) pathway genes in lung tissue from over 1100 subjects from three independent cohorts: the Lung Genomics Research Consortium (LGRC), the Ohio State University (OSU) cohort and the Lung expression quantitative trait loci (Lung eQTL) cohort. These cohorts included patients with Global Initiative for Obstructive Lung Disease (GOLD) stages 1-4 excluding GOLD stage 3. 
Gene expression was normalised and adjusted for age, smoking status (current, former, never), and sex in the LGRC and Lung eQTL study, but not in the OSU study due to sample size. SAULER et al. [16] compared the expression of these genes between very severe COPD (GOLD 4) versus non-severe disease (GOLD 1, 2) and very severe COPD (GOLD 4) versus control (GOLD 0). They reported differential expression of 18 genes in very severe COPD at a false discovery rate $<0.1$ and 15 of these were confirmed using RNA-sequencing and differential display reverse transcription (DDRT)-PCR technique in a subset of the LGRC cohort.

K-means clustering on these 15 genes in all LGRC patients with COPD (GOLD 1-4) identified three clusters which possessed distinct clinical characteristics. Patients in cluster $1(n=65)$ had milder disease that, as clinically expected, was characterised by less pulmonary emphysema and less impairment of diffusing capacity of the lung for carbon monoxide and highest lung function (forced expiratory volume in $1 \mathrm{~s}$ ), exercise tolerance (as measured by the 6-min walk test), quality of life (St George's Respiratory Questionnaire scores) and BODE (body mass index, airflow obstruction, dyspnoea, exercise capacity) index. Whereas no clinical/functional/radiological and/or pathological features distinguished the more severe patients in cluster 2 and cluster 3. Interestingly, there were no differences in sex, pack-years, race or average age across the three clusters.

Three bioinformatic approaches (Gene Set Enrichment Analysis, Z-score and Weighted Gene Correlation Network analysis) were then applied to identify transcriptional changes of known DNA damage and repair pathways that correlated with disease severity. The study found that the mismatch repair, NER, homologous recombination, non-homologous end-joining and Fanconi anaemia pathways were inversely correlated (i.e. protective) with COPD severity to different extents. In contrast, only the direct repair pathway was correlated positively with severity.

Weighted Gene Correlation Network analysis identified 40 modules of co-expressed genes of which several were correlated with disease severity. The advantage of Weighted Gene Correlation Network analysis is that it correlates all the genes that associate with a specific clinical trait however small the difference, so pathways where all the genes have a small difference but these all change in the same direction are given weight as opposed to pathways where only a single gene may change expression. Metacore analysis was used to identify DRR pathways within each module. The yellow module had the strongest negative correlation with measurements of disease severity (pulmonary emphysema, diffusing capacity of the lung for carbon monoxide, forced expiratory volume in $1 \mathrm{~s}, 6$-min walk test, St George's Respiratory Questionnaire and BODE index, all $r>0.4$ and $\mathrm{p}<10^{-7}$ ) and was most enriched for the NER pathway. Specific genes that demonstrated high module membership and gene significance for clinical indices of COPD severity included Xeroderma Pigmentosum Group A Complementing Protein $(x p a)$ and Excision Repair Cross-Complementation Group 5 (ercc5). These three approaches together indicated that downregulation of the NER pathway was highly associated with COPD severity. This emphasises the key role that diminished DNA repair may play in the pathogenesis of COPD.

SAULER et al. [16] took an important step and examined the protein expression of several differentially expressed genes in the three clusters by immunohistochemistry. The expression of DNA damage and repair genes associated with each cluster was highest in the bronchial/bronchiolar epithelium, endothelium and alveolar macrophages. Although the small number of samples analysed $(n=5)$ meant that not all differentially expressed genes were confirmed at the protein level, severe COPD cluster 3 patients demonstrated decreased expression of both NER proteins Damage Specific DNA Binding Protein 2 (DDB2) and Nei like DNA glycosylase 1 (NEIL1). In addition, very severe COPD was associated with upregulation of three of the 15 DDRT-PCR genes, growth arrest and DNA damage-inducible protein GADD45 $\alpha$ (GADD45A) and $\beta$ (GADD45B) and the single-stranded DNA-binding protein oligonucleotide/oligosaccharide-binding fold-containing protein 2A (OBFC2A).

Overall, these data have implications for the increased incidence of lung cancer associated with COPD patients as the NER pathway is primarily responsible for detecting and removing bulky DNA adducts [17], such as polycyclic aromatic hydrocarbons caused by environmental mutagens like cigarette smoke, and is therefore essential for protecting against cigarette smoke-driven carcinogenesis. Although NER was the most significant dysregulated DNA damage and repair pathway associated with severe COPD in this study it is likely that other pathways will be important in subtypes of COPD.

Globally, the data highlights the critical importance of the loss of DNA damage and repair pathways in driving COPD and the potential link with the enhanced incidence of lung cancer seen in these patients [18]. Excess DNA damage due to reduced NER will result in cell death, tissue destruction and inflammation which are key factors in COPD [19].

Genome-wide transcriptomic analysis was also used to identify specific non-DRR pathways associated with each cluster. The top enriched pathways for both cluster 1 (interleukin (IL)-1, IL-3, IL-5, IL-6, IL-17 and 
IL-18) and cluster 3 (IL-3, IL-5, IL-10 and IL-17) were related to cytokine signalling. The IL-5 pathway was the top regulated pathway in both clusters but was downregulated in cluster 1 and upregulated in cluster 3. Cluster 3 also showed up-regulation of the nuclear factor- $\kappa \mathrm{B}$ pathway confirming previous data from our group [20]. Together this raises the question as to whether patients in cluster 3 had a greater number of tissue eosinophils and would have a greater response to anti-eosinophil therapies or to inhaled glucocorticoids. This would be an interesting area for future investigation.

In contrast, severe cluster 2 was characterised by upregulation of transforming growth factor- $\beta$ and WNT pathways implicating a greater degree of cell adhesion and cytoskeletal remodelling in patients within this cluster but also a potential link with autoimmunity [21].

Enhanced inflammation and aberrant remodelling of the lower airways are well described mechanisms of COPD pathogenesis [19]. In addition, there are potential intriguing molecular cross-talks between inflammation-autoimmunity and lung carcinogenesis. For example, polycyclic aromatic hydrocarbons are major inducers of lung carcinogenesis and there are serum anti-polycyclic aromatic hydrocarbons antibodies and antibodies to polycyclic aromatic hydrocarbons-DNA adducts [22]. It is possible that future studies may discover that DNA damage has the potential to trigger both lung-oriented autoimmune responses and carcinogenesis adducts explaining both the autoimmune basis of COPD and their potential link with lung cancer $[23,24]$.

The demonstration that distinct subsets of patients stratified according to DNA damage and repair patterns suggests that future studies of COPD sub-phenotyping, pathogenesis and drug responses should include an analysis of DNA damage and repair patterns in addition to inflammatory/autoimmunity and tissue remodelling pathways.

The study had some limitations and indications for further areas of research. The authors were rightly concerned about the impact of lung cancer. However, it could be argued that changes in NER and other DNA damage and repair pathways along with the other non-DNA damage and repair pathways identified may also have implications for lung cancer development and potentially indicate approaches that could reduce incidence. The data also indicates that these changes in DNA damage and repair expression may also be associated with distinct phenotypes of COPD. In addition, because not all DNA damage and repair genes are controlled at the transcriptional level this may explain the failure to replicate some earlier studies where changes in non-homologous end-joining proteins such as Ku70 were observed in COPD [8]. Proteomic analysis of similar lung tissue samples may address these concerns.

DNA damage and repair proteins were predominantly expressed in alveolar macrophages, bronchial brushings and endothelial cells by immunohistochemistry. Analysis of global gene expression has been performed in bronchoalveolar macrophages, bronchial brushings and small airway epithelial cells from COPD patients [25-27]. Is the reduction in NER and other DNA damage and repair pathways amplified in these cell types where the proteins are expressed?

The authors are to be congratulated on using samples from three independent cohorts but validation in separate datasets and/or patient cohorts is essential. For example, is the NER reduced in severe COPD patients with different ethnic backgrounds and are these observed gene and protein differences clinically relevant?

What is the effect of treatment on the expression of these differentially expressed genes and are they predictive of disease progression and/or response to therapy? The use of conditional knockout mice in animal models of COPD may provide greater information on the functionality of the 3 clusters identified and provide answers to some of the questions raised. The application of CRISPR/Cas9 to attenuate gene expression in airway organoid models will also provide further insight in disease models.

In summary, the authors used a sophisticated bioinformatics approach to study DNA damage and repair pathway changes in COPD lung and identified 15 genes associated with disease severity in three independent cohorts of COPD patients. This 15-DNA damage and repair gene signature identified three clinically distinct disease clusters with distinct non-DNA damage and repair pathways associated with differences in inflammatory, particularly IL-5, and remodelling pathways. The reduced expression of the NER pathway in COPD may not only contribute to disease variability but also to the development of lung cancer.

Conflict of interest: None declared.

\section{References}

1 Punturieri A, Szabo E, Croxton TL, et al. Lung cancer and chronic obstructive pulmonary disease: needs and opportunities for integrated research. J Natl Cancer Inst 2009; 101: 554-559. 
2 Global, regional, and national deaths, prevalence, disability-adjusted life years, and years lived with disability for chronic obstructive pulmonary disease and asthma, 1990-2015: a systematic analysis for the Global Burden of Disease Study 2015. Lancet Respir Med 2017; 5: 691-706.

3 Allemani C, Matsuda T, Di Carlo V, et al. Global surveillance of trends in cancer survival 2000-14 (CONCORD-3): analysis of individual records for 37513025 patients diagnosed with one of 18 cancers from 322 population-based registries in 71 countries. Lancet 2018; 391: 1023-1075.

4 Barnes PJ. Senescence in COPD and its comorbidities. Annu Rev Physiol 2017; 79: 517-539.

5 Rodier F, Coppe JP, Patil CK, et al. Persistent DNA damage signalling triggers senescence-associated inflammatory cytokine secretion. Nat Cell Biol 2009; 11: 973-979.

6 Ahmad T, Sundar IK, Tormos AM, et al. Shelterin telomere protection protein 1 reduction causes telomere attrition and cellular senescence via sirtuin 1 deacetylase in chronic obstructive pulmonary disease. Am J Respir Cell Mol Biol 2017; 56: 38-49.

7 Birch J, Anderson RK, Correia-Melo C, et al. DNA damage response at telomeres contributes to lung aging and chronic obstructive pulmonary disease. Am J Physiol Lung Cell Mol Physiol 2015; 309: L1124-L1137.

8 Caramori G, Adcock IM, Casolari P, et al. Unbalanced oxidant-induced DNA damage and repair in COPD: a link towards lung cancer. Thorax 2011; 66: 521-527.

9 da Silva AL, da Rosa HT, Karnopp TE, et al. Evaluation of DNA damage in COPD patients and its correlation with polymorphisms in repair genes. BMC Med Genet 2013; 14: 93.

10 Yang $\mathrm{S}$, Wu H, Zhao J, et al. Feasibility of 8-OHdG formation and hOGG1 induction in PBMCs for assessing oxidative DNA damage in the lung of COPD patients. Respirology 2014; 19: 1183-1190.

11 Xie J, Yang S, Xu Y, et al. XRCC1 Arg194Trp polymorphism and risk of chronic obstructive pulmonary disease. J Huazhong Univ Sci Technol Med Sci 2009; 29: 551-556.

12 Postel-Vinay S, Vanhecke E, Olaussen KA, et al. The potential of exploiting DNA-repair defects for optimizing lung cancer treatment. Nat Rev Clin Oncol 2012; 9: 144-155.

13 Durham AL, Adcock IM. The relationship between COPD and lung cancer. Lung Cancer 2015; 90: 121-127.

14 Ciccia A, Elledge SJ. The DNA damage response: making it safe to play with knives. Mol Cell 2010; 40: 179-204.

15 Jackson SP, Bartek J. The DNA-damage response in human biology and disease. Nature 2009; 461: 1071-1078.

16 Sauler M, Lamontagne M, Finnemore E, et al. The DNA repair transcriptome in severe COPD. Eur Respir J 2018; 52: 1701994.

17 Scharer OD. Nucleotide excision repair in eukaryotes. Cold Spring Harb Perspect Biol 2013; 5: a012609.

18 Ng Kee Kwong F, Nicholson AG, Harrison CL, et al. Is mitochondrial dysfunction a driving mechanism linking COPD to nonsmall cell lung carcinoma? Eur Respir Rev 2017; 26: 170040.

19 Caramori G, Casolari P, Barczyk A, et al. COPD immunopathology. Semin Immunopathol 2016; 38: 497-515.

20 Di Stefano A, Caramori G, Oates T, et al. Increased expression of nuclear factor-kappaB in bronchial biopsies from smokers and patients with COPD. Eur Respir J 2002; 20: 556-563.

21 Di Stefano A, Sangiorgi C, Gnemmi I, et al. TGF- $\beta$ signaling pathways in different compartments of the lower airways of patients with stable COPD. Chest 2018; 153: 851-862.

22 Pauk N, Klimesova S, Kara J, et al. The relevance of monitoring of antibodies against the polycyclic aromatic hydrocarbon (PAH) and PAH-DNA adducts in serum in relation to lung cancer and chronic obstructive pulmonary disease (COPD). Neoplasma 2013; 60: 182-187.

23 Bozinovski S, Vlahos R, Anthony D, et al. COPD and squamous cell lung cancer: aberrant inflammation and immunity is the common link. Br J Pharmacol 2016; 173: 635-648

24 Caramori G, Ruggeri P, Di Stefano A, et al. Autoimmunity and COPD: clinical implications. Chest 2018; 153: $1424-1431$.

25 Obeidat M, Nie Y, Fishbane N, et al. Integrative genomics of emphysema-associated genes reveals potential disease biomarkers. Am J Respir Cell Mol Biol 2017; 57: 411-418.

26 Tasena H, Faiz A, Timens W, et al. microRNA-mRNA regulatory networks underlying chronic mucus hypersecretion in COPD. Eur Respir J 2018; 52: 1701556.

27 Malhotra R, Kurian N, Zhou XH, et al. Altered regulation and expression of genes by BET family of proteins in COPD patients. PLoS One 2017; 12: e0173115. 